Mediterránea Ser. Biol. (1985), n. ${ }^{\circ}$ 8. Págs. 29-45

\title{
CONTRIBUCION AL CONOCIMIENTO DE LA ESFECIDOFAUNA DE LA PROVINCIA DE ALICANTE \\ (Hym. Sphecidae)
}

\author{
por \\ José Tormos Ferrando1 y Ricardo Jiménez Peydro1.
}

\section{RESUMEN}

Se citan 73 especies y subespecies de esfécidos pertenecientes a las subfamilias Sphecinae, Pemphredoninae, Astatinae, Larrinae, Cabroninae, Nyssoninae, y Philanthinae (sensu Bohart y Menke, 1976); de las cuales Prosopigastra orientalis Beaumont, 1947 constituye nueva cita para la Península Ibérica. Se aportan datos sobre ciertos aspectos de la biología de gran parte de las especies.

\section{SUMMARY}

Are cited 73 species and subespecies belonging to subfamilies Sphecinae, Pemphredoninae, Astatinae, Larrinae, Cabroninae, Nyssoninae, y Philanthinae; 1 is new for the faune of the Peninsula Iberian: Prossopigastra orientalis Beaumont, 1947. So is amplified the knowledge about biology and geographical distribution of these insects.

\section{INTRODUCCION}

Hasta el momento, se han venido realizando una serie de artículos y notas sobre la esfecidofauna de la Comunidad Valenciana (TORMOS, 1983), (TORMOS y JIMENEZ, en prensa a y b), (GAYUBO y TORMOS, en prensa) donde se exponen las características botánicas de la

(1) Departamento de Zoología. Facultad de Ciencias Biológicas. Universidad Literaria de Valencia. VALENCIA. 
zona, metodología, sistemática seguida, antecedentes e importancia de este tipo de estudios faunísticos que se están llevando a cabo.

El presente artículo -que es continuación de los anteriormente enumerados- , se basa en el material recolectado durante los años 1981, 82 y 83 en 20 localidades y puntos de muestreo de la provincia de Alicante dándose, junto al listado de las especies recolectadas, datos de gran importancia sobre la biología de los componentes de esta familia de insectos, como son: tipo de terreno y plantas que frecuentan, así como presas de los mismos.

A continuación se da una lista de las localidades y puntos de muestreo junto con su altitud sobre el nivel del mar. Cada uno de los números que las preceden se corresponden con los representados en el mapa 1 .

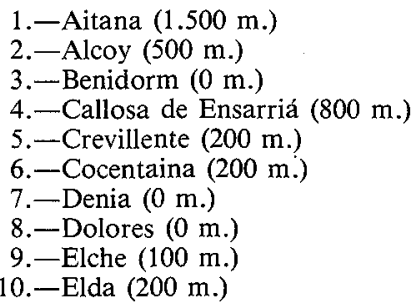

11.-Guardamar $(0 \mathrm{m.})$

12. - Jijona (500 m.)

13.-Monóvar $(200 \mathrm{~m}$.)

14. - Novelda $(200 \mathrm{~m}$.)

15.-Orihuela $(0 \mathrm{~m}$.)

16. - Pego (100 m.)

17. - Santa Faz $(0 \mathrm{~m}$.)

18. -Torrevieja (0 m.)

19.-Villajoyosa $(0 \mathrm{~m}$.)

20. - Villena $(500 \mathrm{~m}$.)

\section{RELACION DE ESPECIES}

Por ahorro de espacio, las localidades se indican sólo por su número de orden.

SPHECINAE

SCELIPHRONINI

Género Chalybion Dahlbom, 1843.

Chalybion (Chalybion) targionii (Caruccio, 1872).

Localidad: 16, V-81, 1 ๙ $^{*}$ sobre Mentha suaveolens Ehrh.

Chalybion (Hemichalybion) femoratum (Fabricius, 1781).

Localidad: 16, VII-81, 1 ơ. Sobre Thapsia villosa L. 
Género Sceliphron Klug, 1801.

Sceliphron (Sceliphron) destillatorium (Illiger, 1807).

Localidades: 5, VII-81, $1 \%$ \%, VIII-82, 7 ९. Sobre Foeniculum vulgare Miller.

Sceliphron (Sceliphron) madraspatanum tubifex (Latreille, 1809).

Localidad: 2, VIII-81, 2 @ ..En zona húmeda.

Sceliphron (Sceliphron) spirifex (Linnaeus, 1758).

Localidad: 2, VII-82, 3 ơ on, 2 ९ ९. En zona húmeda.

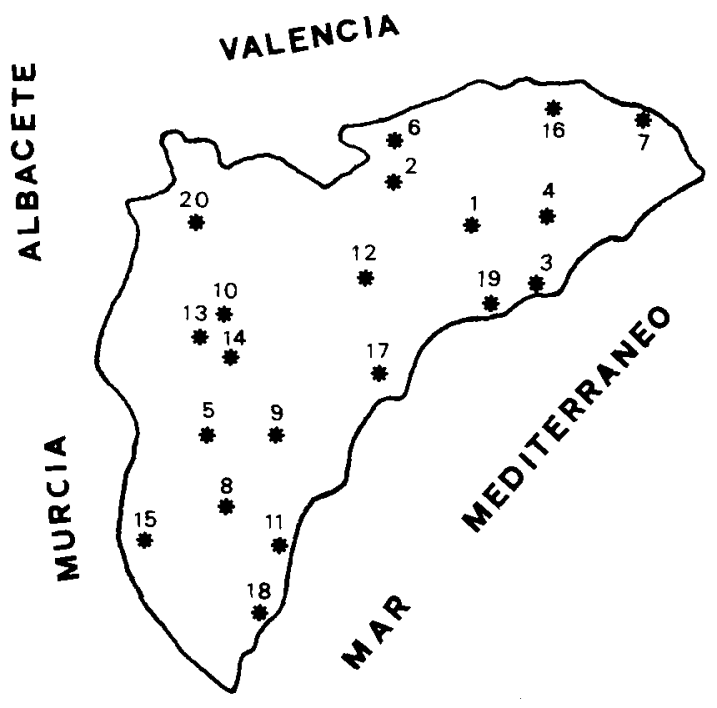

Fig. 1.-Localidades y puntos de muestreo en la provincia de Alicante. 


\section{SPHECINI}

Género Sphex Linnaeus, 1758.

Sphex (Sphex) pruinosus Germar, 1817.

Localidades: 4, V-81, 2 \% ; VIII-81, 3 ᄋ . 19, VII-81, 6 ㅇ . 20, VIII-81, 1 ơ 12, IX-81, 2 ㅇ. 16, VI-82, 1 ; VII-82, 3 우 ; VIII-83, 2 우. 1, VII-82, 1 ơ. 2, VI-83, 1 o . 13. VI-82, 1 ․ 10,

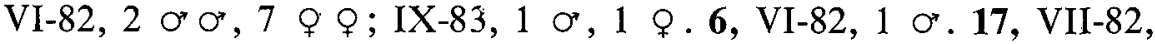
I $\sigma^{\prime} .18$, VII-82, 1 o . 5, VIII-82, 2 o o . 15, VII-83, 3 o o , 2 \% .

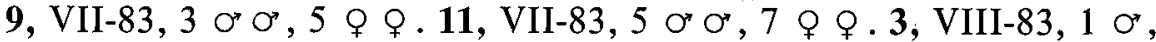

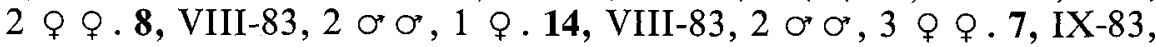
$1 \sigma^{\prime}, 1$. Especie al parecer bastante abundante en la zona de estudio, habiéndose localizado hasta los $1.500 \mathrm{~m}$. de altitud. Se recolectaron ejemplares sobre Brachypodium phoenicoidea (L.) R \& Sch., Pipthatherum miliaceum (L.) Cosson y Stipa tenacissima L., así como en terreno arenoso, arcilloso y arcilloso-pedregoso.

Sphex (Sphex) rufocinctus Brullé, 1883.

Localidades: 4, VII-81, 2 ơ ơ , 5 १ ९; VI-82, 1 ○ , 1 ○ . 14, VIII-83, 2 ơ ơ, 3 ๆ ․ 11, VII-83, 7 or ơ. En cuanto a capturas, idem especie anterior.

Género Prionyx Vander Linden, 1827.

Prionyx kirbyi (Vander Linden, 1827).

Localidades: 1, VI-81, 1 ○, 3 ㅇ . 4, VI-82, 1 ○, 2 ㅇ \&; VII-81,

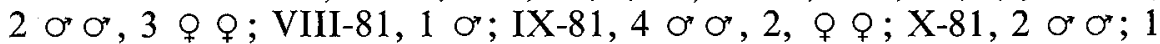

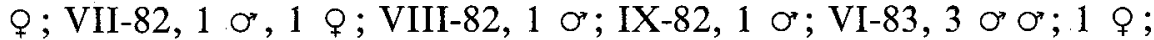

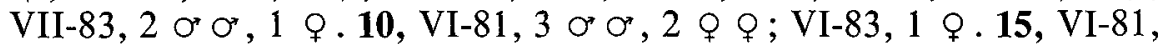
1 ‥ 6, VII-83, 1 o , 1 ‥9, VII-81, 3 o ơ, 1 ㅇ. 11, VII-81, 3 우;

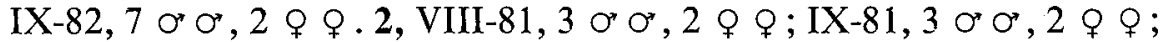
VII-82, 7 우 ․ 5, VII-82, 1 ơ, 3 ㅇ․ . 7, VII-82, 3 ơ ণ , 1 ㅇ. 13, VII-82,

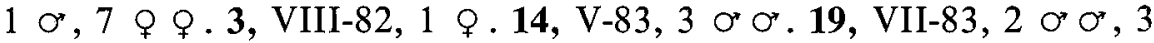
ㅇ. 20, VII-83, 2 o $^{\prime}, 3$ ㅇ․ no arenoso, arcilloso-pedregoso y pedregoso, y sobre las siguientes euphorbias, gramíneas y rutáceas: Euphorbia characias L., Hyparrhenia hirta (L.) Stapf., Piptatherum miliaceum (L.) Cosson Brachypodium retusum (Pers) Beauv. y Ruta angustifolia Pers. Se capturaron hembras con presas de Acrididae (Oedipodinae). 
Prionyx subfuscatus (Dahlbom, 1845).

Localidades: 2, V-82, 1 \% .

\section{AMMOPHILINI}

Género Podalonia, Fernald, 1927.

Podalonia affinis (Kirby, 1798).

Localidades: 14, VII-81, 1 \%

Podalonia hirsuta (Scopoli, 1763).

Localidades: 1, VII-81, 2 \% .2 , XI-81, 1 \% VII-83, 4 ơ $O^{*}, 4$

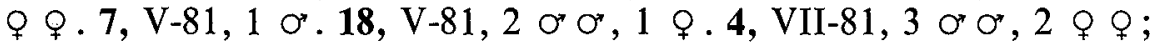

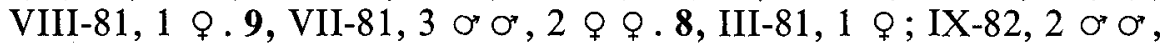
3 o 우 ; V-83, 1 \&. 6, VI-82, 13 ơ Ơ. 5, VII-82, 1 ơ 1 \%; VIII-82,

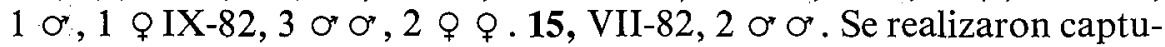
ras sobre terreno arcilloso, arcilloso-pedregoso y pedregoso y sobre las siguientes gramíneas y euphorbias: Brachypodium phoenicoides (L.) R \& Sch., Euphorbia characias L. y Euphorbia helioscopia L.

Se capturaron ejemplares con presa de Lepidoptera Noctuidae.

Podalonia tydei senilis (Dahlbom, 1843).

Localidades: 16, VI-83, 2 \& $९ .3$, V-82, 1 ○ 1 \% . 10, VII-81, 5

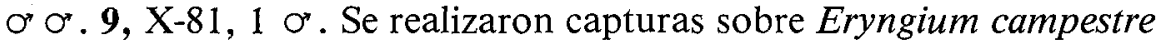
L. y Euphorbia serrata L.

Género Ammophila W. Kirby, 1798.

Ammophila heydeni Dahlbom, 1845.

Localidades: 4, III-81, 2 or on, V-81, 1 ơ 1 ○; VII-82, 1 ㅇ. 16,

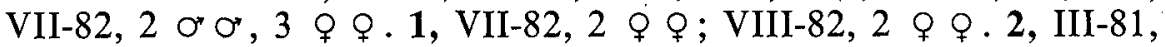
1 Ơ; IV-81, 2 O" Ơ; VIII-83, 3 \& ․

Ammophila hungarica Mocsáry, 1883.

Localidades: 2, V-83, 3 \% . Una de las hembras se capturó con presa de Lepidoptera Pieridae. 
Ammophila laevicollis André, 1836.

Localidades: 4, VI-81, 3 \% ; VII-81, 2 ơ o , 1 \%; VIII-81, 1 o';

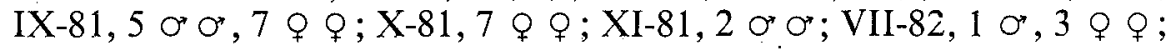

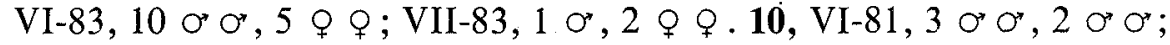
VI-83, 5 or ơ, 10 ᄋ \%; VII-83, 6 ơ ơ; IX-83, 3 \% \%; XI-83, 6 or or,

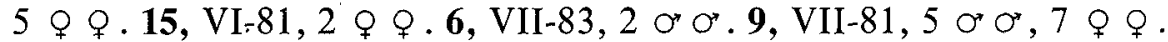
11, VII-81, 2 ㅇ ; IX-82, 8 ơ o*, 5 ㅇ . 2, VIII-81, 1 ; IX-81, 2

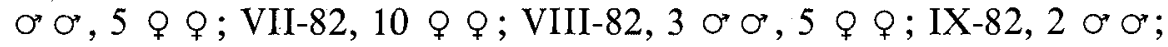

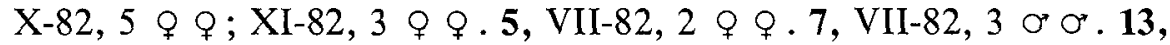
VII-82, 1 o", 1 ᄋ. 3, VIII-82, 1 \&. 14, V-83, 3 on o*. 19, VII-83, 2 or on, 3 \% $९$. 20, VII-83, $10 \%$ \% Se realizaron capturas de imagos en vuelo durante el mes de noviembre. La totalidad de las capturas se realizaron tanto sobre terreno arenoso, arcilloso-pedregoso y pedregoso, como sobre las siguientes gramíneas y umbelíferas Stipa tenacissima L., Brachypodium retusum (Pers) Beauv., Foeniculum vulgare Miller y Daucus carota $\mathrm{L}$.

Ammophila pubescens Curtis, 1836.

Localidad: 7, VII-82, 1 \%, 1 \&.

Ammophila sabulosa (Linnaeus, 1758).

Localidades: 4, VI-81, 1 \% ; VII-81, 1 or; VIII-81, 3 or or; IX-81,

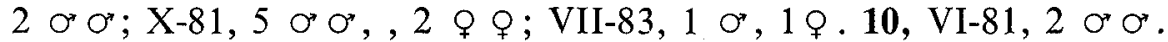

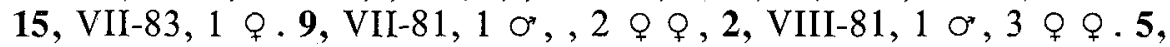
VII-82, 2 ○

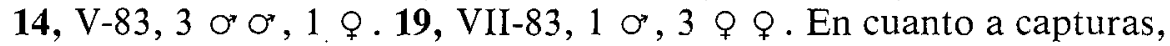
idem. Ammophila laevicollis André.

\section{PEMPHREDONINAE}

\section{PEMPHREDONINI}

Género Diodontus Curtis, 1834.

Diodontus luperus Shuckard, 1837.

Localidades: 4, III-81, 2 o $0^{\circ}, \mathrm{V}-81,1$ o , 1 \%; VII-82, 1 \%. 1,

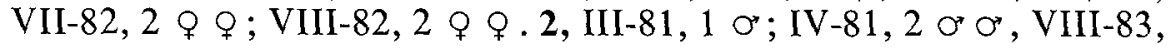
3 $\subsetneq$. Se capturaron ejemplares cuando volaban alrededor de diver- 
sas euforbias y umbelíferas muy atacadas por Aphis sp. y Sitobion sp. (Homoptera, Aphoidea).

Género Pemphredon Latreille, 1796.

Pemphredon lethifer Shuckard, 1837).

Localidades: 14, VII-81, 1 or; 11, VII-82, 2 ○.

ASTATINAE

ASTATINI

Género Astata Latreille, 1796.

Astata boops boops (Schrank, 1781).

Localidad: 11, VII-81, 2 on on, 1 @. Sobre terreno arenoso.

Astata boops picea Costa, 1867.

Localidad: 3, VIII-82, 1 \&. Sobre terreno arenoso.

Astata minor Kohl, 1885.

Localidad: 3, VIII-82, 1 ․ Sobre terreno arenoso.

LARRINAE

LARRINI

Género Larra Fabricius, 1793.

Larra (Larra) anathema (Rossi, 1790).

Localidad: 16, VI-82, 3 ơ $\mathrm{O}^{\prime}, 2$ \% $\mathrm{Q}$. Sobre terreno pedregoso. Género Liris Fabricius, 1804.

Liris (Leptolarra) atrata Spinola, 1805).

Localidad: 7, VII-82, 5 ○ $\circlearrowleft, 7$ ९ . Sobre terreno arcilloso. Liris (Leptolarra) nigra (Fabricius, 1775). 
Localidades: 16, VIII-81, 1 \%.3, VII-82, 2 ९ . 11, VII-83, 3 ९ . Sobre terreno arenoso y arcilloso.

Liris (Leptolarra) praetermissa (Richards, 1928).

Localidad: 7, VII-82, 3 \& . Sobre terreno arcilloso.

Género Tachytes Panzaer, 1806.

Tachytes europaeus Kohl, 1884.

Localidad: 16, VII-81, 1 ơ, 1 ๆ. Sobre Mentha sp.

Género Tachysphex Kohl, 1883.

Tachysphex erythropus (Spinola, 1838).

Localidad: 8, VIII-81, 1 .

Tachysphex fugax (Radoszkowski, 1877).

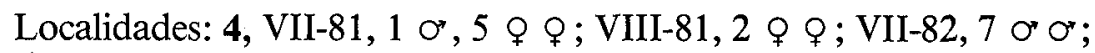

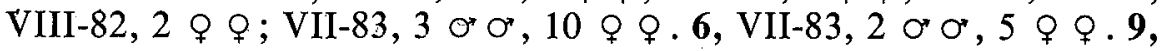

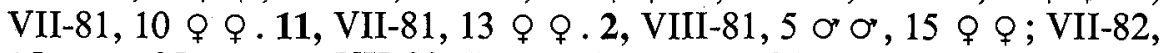

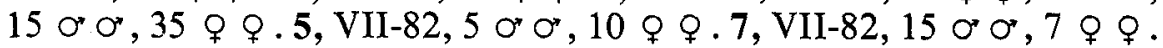

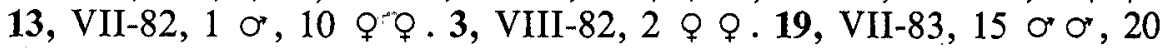

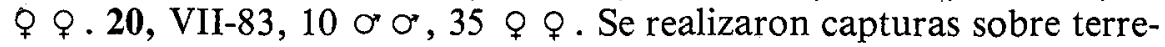
no arenoso, arcilloso, arcilloso-pedregoso y pedregoso, así como sobre Mentha suaveolens Ehrh.

Tachysphex incertus (Radoszkowski, 1877).

Localidades: 4, VII-81, 2 o* or; VIII-81, 1 \%; VII-82, 3 ९ ; VIII-82, 1 \% . 6, VII-83, 1 ๆ. 9, VII-81, 3 ९ $९ .11$, VII-81, 1 ○ . 2, VIII-81, $1 \circ^{*}, 2$ \% $q$ VII-82, $1 \circ^{\circ} .20$, VIII-83, 1 ơ $^{*}$ En cuanto a capturas idem. especie anterior.

Tachysphex mediterraneus (Kohl, 1883).

Localidades: 4, VII-81, 1 o ; VII-82, 2 ९ $९$; VIII-82, 1 o . 9, VII-81, 1 o ;1, VII-81, 1 \&. Se realizaron capturas sobre terreno arenoso y arcilloso. 
Tachysphex nitidus (Spinola, 1805).

Localidades: 11, VII-81, 1 .

Tachysphex panzeri (Vander Linden, 1829).

Localidades: 4, VII-81, 1 ơ; VIII-81, 2 \% ; VII-82, 3 \% $\%$;

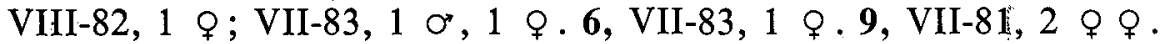

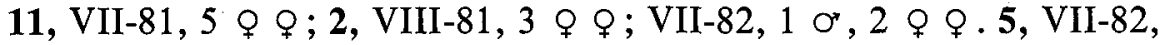
$1 \sigma^{\circ}, 3$ ㅇ . . 7, VII-82, 2 ๑

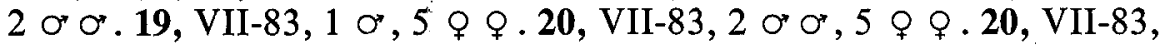
1 ơ, 1 ㅇ. Se realizaron algunas capturas sobre Mentha suaveolens Ehrh.

Tachysphex obscuripennis (Schenck, 1857).

Localidad: 16, VII-81, 1 ९. Sobre terreno arcilloso-pedregoso.

Tachysphex pompiliformis (Panzer, 1804).

Localidad: 16, VIII-81, 1 q . La captura se realizó cuando el ejemplar volaba alrededor de Eryngium campestre L.

Género Prosopigastra Costa, 1867.

Prosopigastra orientalis Beaumont, 1947.

Localidades: '11, VII-81, 2 ○' ơ. Sobre terreno arenoso.

Especie que se cita por primera vez para la Península Ibérica. En el mapa 2 se refleja su distribución geográfica según (PULAWSKI, 1979) así como la nueva cita.

Prosopigastra punctatissima Costa, 1867.

Localidades: 19, VII-81, 1 o $^{*}$ 11, VII-81, 1 ․ 3, VII-82, 3 ㅇ. Sobre terrenó arenơso.

\section{MISCOPHINI}

Género Miscophus Jurine, 1807.

Miscophus eatoni E. Saunders, 1903.

Localidad: 11, VII-81, 1 ㅇ. Sobre terreno arenoso. 


\section{TRYPOXYLONINI}

Género Trypoxylon Latreille, 1796.

Trypoxylon (Trypoxylon) figulus (Linnaeus, 1758).

Localidad: 19, VIII-82, 2 ○. Sobre Euphorbia helioscopia L.

\section{CABRONINAE}

OXYBELINI

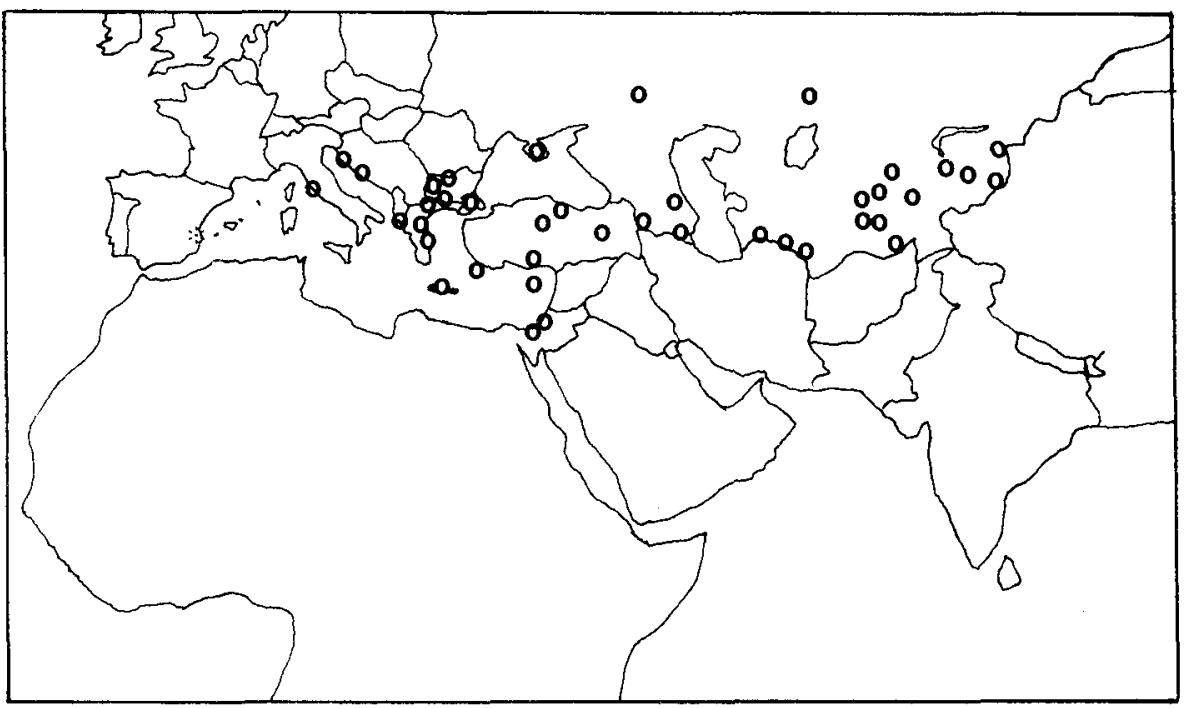

Fig. 2.-Distribución geográfica de Prosopigastra orientalis. Beaumont, 1947; o, citas anteriores*, *nueva cita.

Género Oxybelus Latreille, 1796.

Oxybelus andalusiacus Spinola, 1843.

Localidades: 3, VI-81, 2 o 11, VI-82, 3 \% $९$; VHI-82, 2 ○

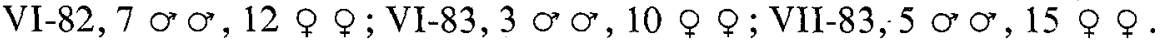

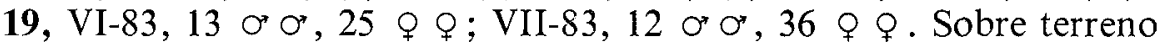
arenoso. 
Según NINGO (1969), los datos biológicos conocidos de esta especie son muy poco numerosos. Se capturó una hembra con presa de Diptera Muscidae.

Oxybelus dissectus Dahlbom, 1845.

Localidad: 3, VII-82, 1 q. Sobre Dittrichia viscosa (L.) N. Grenter. Oxybelus latro Olivier, 1811.

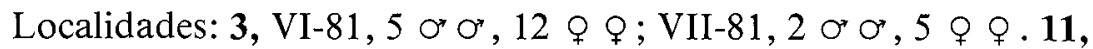

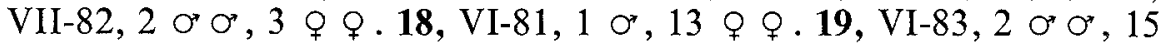
ㅇ ; VII-83, 3 ơ $\mathrm{O}^{*}, 17$ ㅇ . Sobre terreno arenoso.

Oxybelus mucronatus (Fabricius, 1793).

Localidades: 3, VI-81, 3 or ơ; VII-81, 10 ơ ơ, 22 ๆ $~ .11$, VI-82,

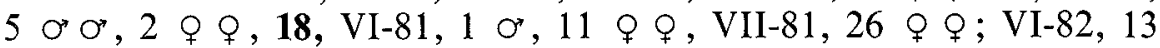
or ơ, 2 ㅇ ; VII-82, 25 ㅇ ․ 19, VI-83, 13 or or, 27 우. Se realizaron capturas tanto sobre terreno arenoso como sobre Euphorbia helioscopia $\mathrm{L}$.

Oxybelus subspinosus Klug, 1835.

Localidad: 3, VII-82, 1 \%, 2 $\subsetneq$. Sobre terreno arenoso.

Oxybelus trispinosus (Fabricius, 1787).

Localidad: 11, VII-83, 2 $~$. La captura se efectuó cuando el ejemplar volaba alrededor de Euphorbia serrata L.

Oxybelus victor Lepeletier, 1845.

Localidades: 3, VI-81, 2 ơ Or; VII-81, 1 ơ 4 \% $\%$. 11, VI-82, 2

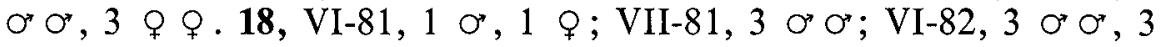

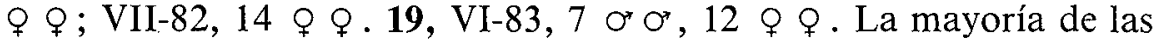
capturas se realizaron sobre terreno arenoso.

Se capturó una hembra con presa de Diptera Sarcophagidae. CABRONINI

Género Lindenius Lepeletier y Brullé, 1834. 
Lindenius pygmaeus pygmaeus (Rossi, 1794).

Localidad: 11, VI-82, 1 ơ. Sobre terreno arenoso.

Género Crossocerus Lepeletier y Brullé, 1834.

Crossocerus palmipes (Linnaeus, 1767).

Localidades: 6, V-81, 1 o* 1 \%. 16, V-81, 2 \% . Se realizó una captura alrededor de Anacylus valentinus L.

Género Lestica Billberg, 1820.

Lestica clypeata (Schreeber, 1759).

Localidades: 2, VI-81, $1 \circ^{*}, 2$ ᄋ $\%$; VII-81, 3 or $0^{*}$; VII-83, 3 or $0^{*}$.

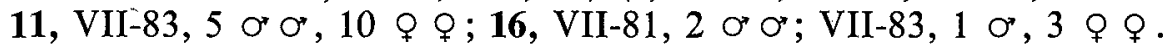
Sobre terreno arcilloso, arcilloso-pedregoso y pedregoso.

\section{NYSSONINAE}

GORYTINI

Género Gorytes Latreille, 1804.

Gorytes sulcifrons (Costa, 1869).

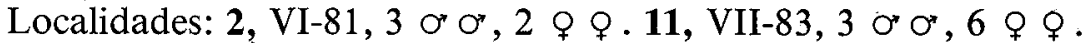
Se realizaron capturas sobre Centaurea aspera L. y Euphorbia helioscopia L.

Género Sphecius Dahlbom, 1843.

Sphecius nigrocornis (Dufour, 1838).

Localidades: 3, VI-81, 3 ơ ơ. 18, VIII-83, 2 ९ . 19, VIII-83, 5 $0^{\circ} \odot^{*}, 2$ \% . Sobre terreno arenoso.

\section{STIZINI}

Género Stizus Latreille, 1802.

Stizus continuus Klug, 1835). 


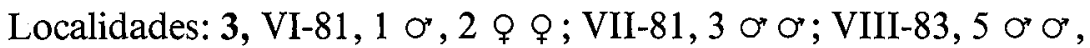

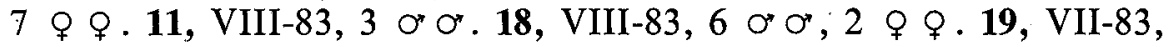
2 ơ ơ; VIII-83, 3 \& $\%$. Sobre terreno arenoso.

Stizus ruficornis (Forster, 1771).

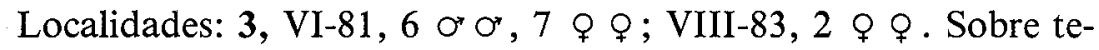
rreno arenoso.

\section{BEMBECINI}

Género Bembix Fabricius, 1775.

Bembix bidentata Vander Linden, 1829.

Localidad: 3, VI-81, 1 q. Sobre terreno arenoso.

Bembix flavescens bolivari Handlirsch, 1893.

Localidades: 3, VI-81, 2 on ơ 3 \% ९; VII-81, 2 \% ; VI-82, 5 on on;

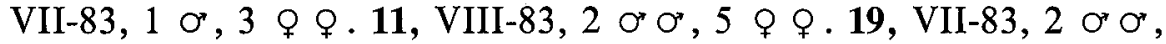
1 \%; VIII-83, 2 \% $\odot$. Se capturaron ejemplares sobre terreno arenoso y Eryngium campestre L.

Bembix oculata Panzer, 1801.

Localidad: 3, VII-83, 6 ơ ơ, 9 ㅇ $९$. Sobre terreno arenoso.

Se capturó una hembra con presa de Diptera (Bombilidae).

Bembix rostrata (Linnaeus, 1758).

Localidad: 3, VII-83, 1 q. Sobre terreno arenoso.

PHILANTHINAE

PHILANTHINI

Género Philanthus Fabricius, 1790.

Philanthus coronatus ibericus Beaumont, 1970.

Localidad: 16, VII-83, 2 ơ ơ, 3 ㅇ. 
Philanthus dufourii Lucas, 1848.

Localidad: 16, VII-83, 2 ๆ .

Philanthus pulchellus Spinola, 1842.

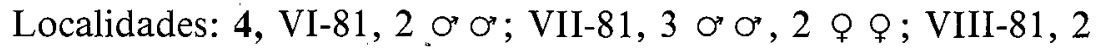

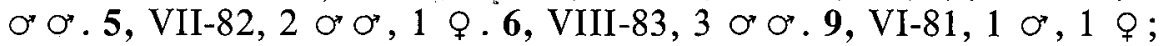

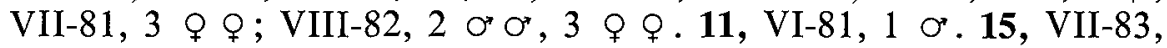

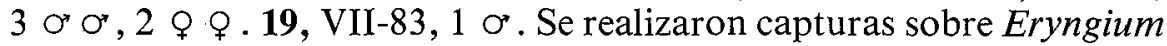
campestre L. y Thapsia villosa $\mathrm{L}$.

Philanthus triangulum (Fabricius, 1775).

Localidades: 2, V-81, 3 ơ $\odot, 2$ ㅇ ९; VI-81, 6 ○ ० 7 ९ ९; VII-81,

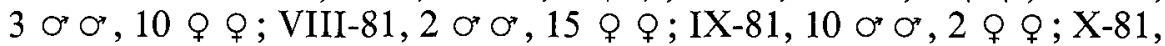

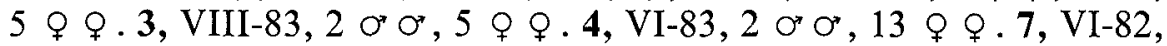

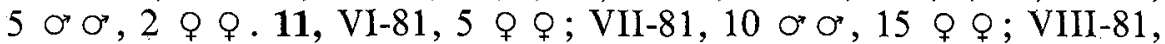

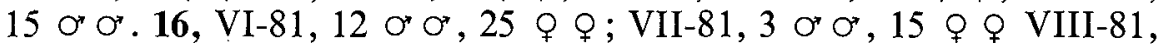

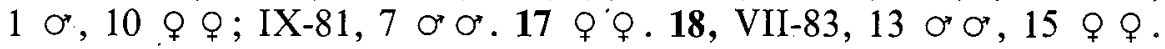

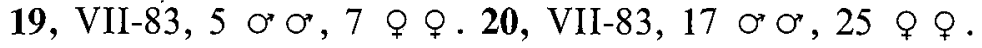

La gran mayoría de las capturas se realizaron sobre las siguientes umbelíferas: Eryngium campestre L., Foeniculum vulgare Miller y Thapsia villosa $\mathrm{L}$.

Aunque las presas más conocidas de esta especie de esfécido son individuos pertenecientes a la especie Apis mellifera L., ocasionalmente suelen atacar a abejas solitarias pertenecientes a los géneros Andrena, Dasypoda, Megachile y Halictus. BEEKHUIS VAN TILL (1935).

Se capturaron tres hembras con presas de Halictus sp.

\section{CERCERINI}

Género Cerceris Latreille, 1802.

Cerceris arenaria (Linnaeus, 1758).

Localidades: 3, VI-81, 3 or ơ 2 $\%$. Sobre terreno arenoso. Cerceris bellona Mercet, 1914.

Localidad: 3, VI-81, 1 ○’, 2 ९ . Sobre terreno arenoso. 
Cerceris boetica Pérez, 1913.

Localidad: 3, VI-81, 2 ơ ox, 3 우․ Sobre terreno arenoso.

Cerceris bupresticida Dufour, 1841.

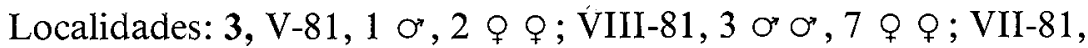

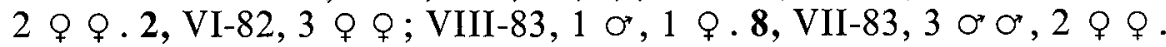
9, VII-83, 2 우. 11, VI-81, 5 o o , 3 ㅇ; ; VII-81, 13 o 16, VIII-83, 5 ơ o , 3 ㅇ . 20, VIII-83, 5 or o , 17 우; IX-83, 2 ㅇ . Las capturas se realizaron sobre terreno arenoso y pedregoso; Euphorbia helioscopia L. y Euphorbia serrata L.

Cerceris lunata funerea A. Costa, 1869.

Localidades: 18, VII-83, 2 or ๙

Cerceris moroderi Giner Marí, 1941.

Localidades: 3, V-81, 2 ○ ơ, 5 ㅇ . 20, VIII-83, 1 ○, 2 \& ㅇ. . Los ejemplares pertenecientes a la primera localidad se capturaron en su totalidad sobre terreno arenoso.

Cerceris quadricincta (Panzer, 1799).

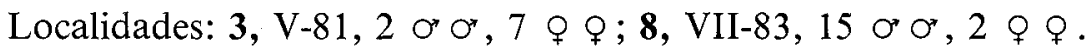
Sobre terreno arenoso.

Cerceris rybyensis (Linnaeus, 1771).

Localidades: 3, V-81, 3 or o , 15 \% ; VI-81, 5 o o , 25 q ;

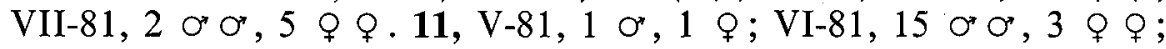
VII-81, 1 ণ, 2 ㅇ․ 17, VI-82, 13 ơ ơ, 25 우 . 18, VII-83, 1 ơ . 19, VII-83, 2 ○ $0^{*}, 1$ q. Sobre terreno arenoso.

Cerceris sabulosa (Panzer, 1799).

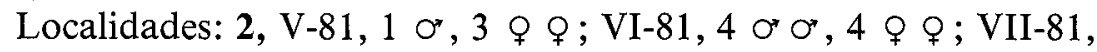

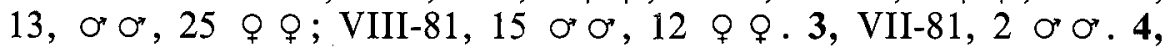

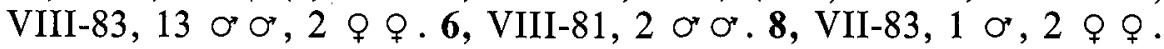

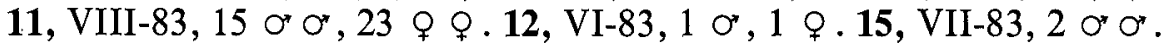

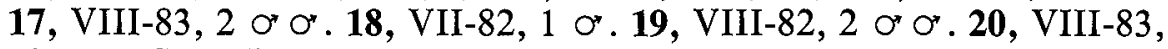
$13 q q$. Se realizaron capturas sobre terreno arenoso, arcilloso, arcilloso- 
pedregoso y pedregoso, así como sobre Euphorbia peplis L., Mentha sp., Medicago marina L. y Stipa tenacissima L.

Se capturaron algunas hembras con presas de Halictus sp.

Cerceris specularia Costa, 1869.

Localidad: 9, VII-83, 1 \% .

Cerceris tuberculata (Villers, 1789).

Localidades: 2, V-81, 2 \% . 15, VIII-83, 3 ९ . 


\section{BIBLIOGRAFIA}

BOHART, R.M. y A.S. MENKE, (1976). Sphecid wasps of the world. A generic revision. University of California Press. Berkeley. 695 págs.

GAYUBO, S.F. y TORMOS, (en prensa). Nuevas aportaciones al conocimiento de la esfecidofauna valenciana (Hym., Sphecidae). «Fundación entomológica Juan de Torres Sala».

MINGO PEREZ, E., (1966). Los Oxybelini de la Península Ibérica. Graellsia, XXII: 57-123.

PULAWSKI, W.J.,.(1976). A revision of the world Prosopigastra Costa (Hymenoptera, Sphecidae). Polsk. Pis. Entomol., 49: 3-134.

SIMON THOMAS; R.T. y A.M.J. SIMON THOMAS, (1972). Some observations on the Behaviour of females of Philanthus triangulum (F.) (Hymenoptera, Sphecidae). Tijdschr. Ent. 115: $123-139$.

TORMOS, J., (1983). Nueva cita de Passaloeus corniger Shuckard, 1837 en la Península Ibérica (Hym., Sphecidae). Bol. Asoc. Esp. Entom., 7: 321.

TORMOS, J. y R. JIMENEZ, (en prensa a). Esfécidos de la Provincia de Valencia (Hymenoptera, Sphecoidea). Nouv. Rev. Ent.

TORMOS, J. y R. JIMENEZ, (en prensa b). Estudio comparativo de la esfecidofauna de la Dehesa del Saler (Hym., Sphecoidea). Eos. 